\title{
Evidence that the degree of band 3 phosphorylation modulates human erythrocytes nitric oxide efflux - in vitro model of hyperfibrinogenemia
}

\author{
J.P. Lopes de Almeida ${ }^{\mathrm{a}, \mathrm{b}, *}$, T. Freitas-Santos ${ }^{\mathrm{b}}$ and C. Saldanha ${ }^{\mathrm{a}, \mathrm{b}}$ \\ ${ }^{a}$ University of Lisbon Medical School, Santa Maria Hospital, Lisbon, Portugal \\ ${ }^{\mathrm{b}}$ Institute of Biochemistry, Institute of Molecular Medicine, University of Lisbon Medical School, \\ Lisbon, Portugal
}

\begin{abstract}
Recent evidence has shown that plasma fibrinogen, a major cardiovascular risk factor, interacts with the erythrocyte membrane and acts to influence blood flow via erythrocyte nitric oxide (NO) modulation. In the present pioneer in-vitro study, whole blood samples were harvested from healthy subjects and aliquots were incubated in the absence (control aliquots) and presence of fibrinogen at different degrees of band 3 phosphorylation, and the levels of NO, nitrite, nitrate and S-nitroglutathione (GSNO) were determined.

Hyperfibrinogenemia interferes with erythrocyte NO mobilization without changing its efflux in a way that seems to be dependent of the degree of band 3 phosphorylation. In presence of higher fibrinogen concentrations the NO efflux is reinforced when band 3 is phosphorylated $(p<0.001)$. Higher levels of nitrite, nitrate and GSNO were documented $(p<0.05)$. However, the mechanisms by which fibrinogen signalling modulates erythrocyte function remain to be clarified and are currently under study. These conditions may be considered an approach to be followed in blood storage for transfusions.
\end{abstract}

Keywords: Band 3 protein, erythrocyte, fibrinogen, nitric oxide, nitrates, nitrites, S-nitrosoglutathione

$\begin{array}{ll}\text { Abbreviations } \\ \text { GSNO } & \text { S-nitrosoglutathione } \\ \mathrm{NO} & \text { Nitric oxide } \\ \mathrm{NOx} & \text { Nitric oxide metabolites } \\ \mathrm{NO}_{2}^{-} & \text {Nitrites } \\ \mathrm{NO}_{3}^{-} & \text {Nitrates }\end{array}$

${ }^{*}$ Corresponding author: J. Pedro Lopes de Almeida, Instituto de Bioquímica, Instituto de Medicina Molecular, Faculdade de Medicina de Lisboa, Edifício Egas Moniz, Av. Prof. Egas Moniz, 1649-028 Lisboa, Portugal. Tel.: +351 91 8985450; Fax: +351 21 7999477; E-mail: jpedro.gla@gmail.com. 


\section{Introduction}

Vascular nitric oxide (NO) traverses red blood cells by simple random diffusion and it may either be stored or return to the bloodstream as an active S-nitrosothiol molecule. It interacts with several components of the erythrocyte membrane, as well as with hemoglobin [16, 27, 28]. The major stable metabolites resulting from $\mathrm{NO}$ oxidation, represented by $\mathrm{NOx}$, include nitrite $\left(\mathrm{NO}_{2-}\right)$ and nitrate $\left(\mathrm{NO}_{3-}\right)$. Glutathione is an antioxidant molecule with a thiol group which binds to NO yielding S-nitrosothiols (GSNO), another molecular form of NO storage [38].

Vascular NO has been identified as having a key role in blood pressure regulation, being synthesised by inducible nitric oxide synthase in cases of arterial hypertension, vascular pathologies and inflammation conditions with deleterious effects $[6,40]$. It has not only a key role in blood pressure regulation but also in blood flow regulation $[17,26]$. In addition, according to the literature there is a wide range of other conditions inducing NO secretion. Some examples include different drugs used in cardiovascular medicine (e.g. corticosteroids) as well as during physical activity (e.g. stress test) [5, 11, 23, 30, 39, 42].

Additionally, fibrinogen is a plasma protein involved in haemostasis, cell adhesion and inflammation, also known to behave as a hemorheological factor by promoting the formation of erythrocyte aggregates $[2,15,20,25,29,32]$. The hyperaggregation state induced by fibrinogen takes place in various metabolic and cardiovascular diseases such as diabetes, arterial hypertension and atherosclerosis [21]. Moreover, a linkage between erythrocyte adhesiveness and plasma fibrinogen levels (under inflammatory conditions) has been documented in subjects with atherosclerotic risk factors [34].

In vitro, we have observed a significant increase in erythrocyte GSNO, nitrite and nitrate concentrations in the presence of fibrinogen at physiological levels, when compared with blood samples in the absence of this plasma protein. On the contrary, the erythrocyte NO efflux decreases in the presence of fibrinogen at the same range of concentrations.

Comprising up to $25 \%$ of the erythrocyte membrane, band 3 is a predominant protein and the major mediator of anion transport in the human erythrocyte membrane. It spans the membrane asymmetrically and participates in a number of erythrocyte events regulated by its degree of phosphorylation. Band 3 phosphorylation is promoted by tyrosine-kinases (PTK, e.g. syk and lyn) and dephosphorylation by tyrosine-phosphatases (PTP) [4]. We have documented that band 3 is directly involved in NO efflux and mobilization, by modulation of its phosphorylation degree [7].

Considering the fact that all degrees of inflammation are associated with reactive oxygen and nitrogen species, along with an hyperfibrinogenemia state, we might raise the hypothesis of concentration changes occurring in NO and NO-derived molecules. However, no work has yet assessed the effect of high levels of plasma fibrinogen on the erythrocyte nitric oxide metabolism (nitrite, nitrate) and mobilization (Snitroso-glutathione), on the dependence of the degree of band 3 phosphorylation, which was the purpose of this study.

\section{Methods}

\subsection{Chemicals}

The chosen concentrations for fibrinogen were based on its physiological levels and previous studies [36]. The human fibrinogen was purchased from Sigma (Poole, UK). p72 ${ }^{\text {syk }}$ inhibitor and aminogenistein (p53/56 $6^{\mathrm{yn}}$ inhibitor) were purchased from Sigma (St Louis, MO, USA), and calpeptin (PTP inhibitor) was purchased from Calbiochem (Darmstadt, Germany). 
The study was performed according to the guidelines of Clinical Hemorheology and Microcirculation [1].

\subsection{Experimental design}

Human venous blood samples were collected from the forearm vein of fifteen healthy Caucasian men after informed consent. The blood container tubes were prepared with $10 \mathrm{IU} \mathrm{ml}^{-1}$ of sodium heparin (anticoagulant). After that, the blood was divided in ten $1 \mathrm{~mL}$ aliquots and centrifuged at 11,000 rpm for 1 minute in Biofuge 15 centrifuge (Heraeus, Sepatech, Osterode, DE). $5 \mu \mathrm{L}$ of plasma was removed from one aliquot and the same volume of fibrinogen solution was added. After mixing, other centrifugation step was done, and plasma removed for fibrinogen concentration determination and the same procedure for the control aliquot. From the other four aliquots, $5 \mu \mathrm{L}$ of plasma was taken and replaced by the same volume in order to achieve $10 \mu \mathrm{M}$ final concentration of Syk inhibitor, AMGT and calpeptin. To the remaining four fibrinogen solutions, band 3 effectors were added to achieve the $10 \mu \mathrm{M}$ final concentration of each one. Blood samples aliquots were then incubated during $30 \mathrm{~min}$ at room temperature after slight agitation, in the absence and presence of fibrinogen.

\subsection{Determination of fibrinogen concentration by clot-based technology}

Plasma fibrinogen concentrations were evaluated using the Fibritimer Dade Behring BF TII based in the Clot-based technology.

\subsection{Measurement of NO by an amperometric method}

Following incubation, erythrocyte suspensions were centrifuged and sodium chloride $0.9 \%$ at $\mathrm{pH} 7.0$ was added in order to compose a hematocrit of $0.05 \%$. The suspension was mixed by gently inversion of tubes.

For amperometric NO quantification we used the amino-IV sensor (Innovative Instruments Inc. FL, USA), a method previously described by us [9]. NO diffuses through the gas-permeable membrane tripleCOAT of the sensor probe and it is then oxidized at the working platinum electrode, resulting in an electric current. The redox current is proportional to the NO concentration outside the membrane and is continuously monitorized with an inNO ${ }^{\mathrm{TM}}$ software (version 1.9 from Innovative Instruments Inc., FL, USA) and connected to a computer. Calibration of the NO sensor was performed daily. For each experiment, the NO sensor was immersed vertically in the erythrocyte suspension vials and allowed to stabilize for $30 \mathrm{~min}$ to achieve NO basal levels. $30 \mu \mathrm{l}$ of acetylcholine (ACh, purchased from Sigma St. Louis, MO, USA) was added to the erythrocyte suspensions aliquots in order to achieve the final concentrations of ACh $10 \mu \mathrm{M}$ and the NO released from erythrocytes was registered. Data were recorded from constantly stirred suspensions at room temperature.

\subsection{Measurement of nitrite/nitrate concentration using the spectrophotometric griess method}

After the incubation period the erythrocytes suspensions, performed as described above, were centrifuged at $9600 \mathrm{~g}$ during 1 minute using the Biofuge 15 Heraeus centrifuge. The supernatants were then separated from the pellet (packaged erythrocytes). 
Nitrite and nitrate levels in the intra-erythrocyte compartment were determined, as previously published by us [10], after submitting the pellet of each suspension to hemolysis and hemoglobin precipitation (erythrocyte cytoplasm values registered). Hemolysis was induced with distilled water and hemoglobin precipitation with ethanol and chloroform.

Nitrite concentrations were measured with the spectrophotometric Griess reaction, at $548 \mathrm{~nm}$. For nitrate measurement, this compound was first reduced to nitrites in the presence of nitrate reductase [18].

\subsection{Measurement of S-nitrosoglutathione (GSNO)}

Colorimetric solutions containing a mixture of sulfanilic acid (B component of Griess reagent) and NEDD (A component of Griess reagent), consisting of $57.7 \mathrm{mM}$ of sulfanilic acid and $1 \mathrm{mg} / \mathrm{mL}$ of NEDD, were dissolved in phosphate-buffered solution, $\mathrm{pH} 7.4$ (PBS). To constitute the $10 \mathrm{mM} \mathrm{HgCl}_{2}$ (Aldrich) mercury ion stock solutions were prepared in $0.136 \mathrm{~g} / 50 \mathrm{~mL}$ of dimethyl sulfoxide (DMSO) (Aldrich). GSNO was diluted to the wanted concentration in the colorimetric analysis solutions. Various concentrations of mercury were then added to a final concentration of $100 \mu \mathrm{M}$. Following gentile shaking the solution was let to stand for twenty minutes. A control spectrum was measured by spectrophotometry at $496 \mathrm{~nm}$ against a solution without mercury ion. $300 \mu \mathrm{l}$ of erythrocyte suspensions were added to the reaction mixture and GSNO concentrations were obtained as described by Cook [12].

\subsection{Statistical analysis}

Data are expressed as means \pm SD. Student's paired $t$-tests were used to compare values between different aliquots of erythrocyte suspensions. Statistical analysis was conducted using the Statistical Package for the Social Sciences (SPSS) software, 16.0 version. One-way analysis of variance tests and paired $t$-tests were applied to assess statistical significance amongst samples. Bonferroni post-hoc tests were conducted when appropriate. Statistical significance was set at a $p<0.05$ level.

\section{Results}

\subsection{Effects of in vitro hyperfibrinogenemia and band 3 phosphorylation/dephosphorylation effectors on nitric oxide levels (Fig. 1)}

Statistically significant higher values were found for the erythrocyte NO efflux measured in aliquots incubated with band 3 modulators of its degree of phosphorylation, when compared with the control suspensions, but not under a hyperfibrinogenemia state.

In the presence of high fibrinogen levels (mean \pm SD: $480.6 \pm 114 \mathrm{mg} / \mathrm{dL}$ ) the levels for NO accounted for $1.91 \pm 0.39 \mathrm{nM}$, when compared with the control aliquots without fibrinogen $(1.63 \pm 0.49 \mathrm{nM}$, $p=0.15$.

With band 3 effectors the following NO levels were observed: (i) $2.20 \pm 0.38 \mathrm{nM}$ in the presence of Syk inhibitor $10 \mu \mathrm{M}$ ( $p=0.02$, against control), (ii) $2.11 \pm 0.38 \mathrm{nM}$ in the presence of AMGT $10 \mu \mathrm{M}(p=0.02$, against control), and (iii) $2.35 \pm 0.27 \mathrm{nM}$ in the presence of calpeptin $10 \mu \mathrm{M}(p=0.005$, against control).

In the presence of additional fibrinogen, no significantly changes were observed for the nitric oxide levels, except for band 3 phosphorylation inibitors when compared with those values obtained only with fibrinogen. 


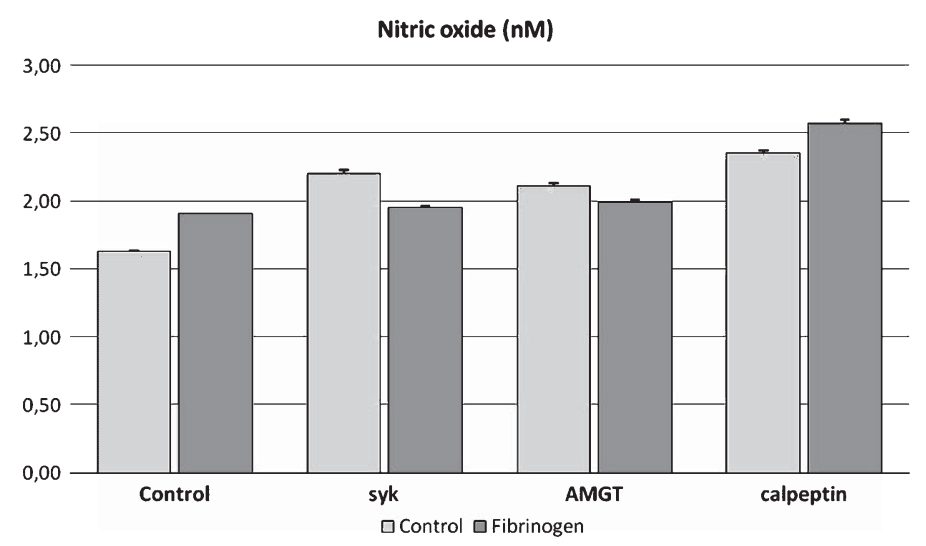

Fig. 1. Effects of high fibrinogen and band 3 modulators on nitric oxide levels in erythrocyte suspensions. Values are mean \pm $\operatorname{SD}(n=15)$.

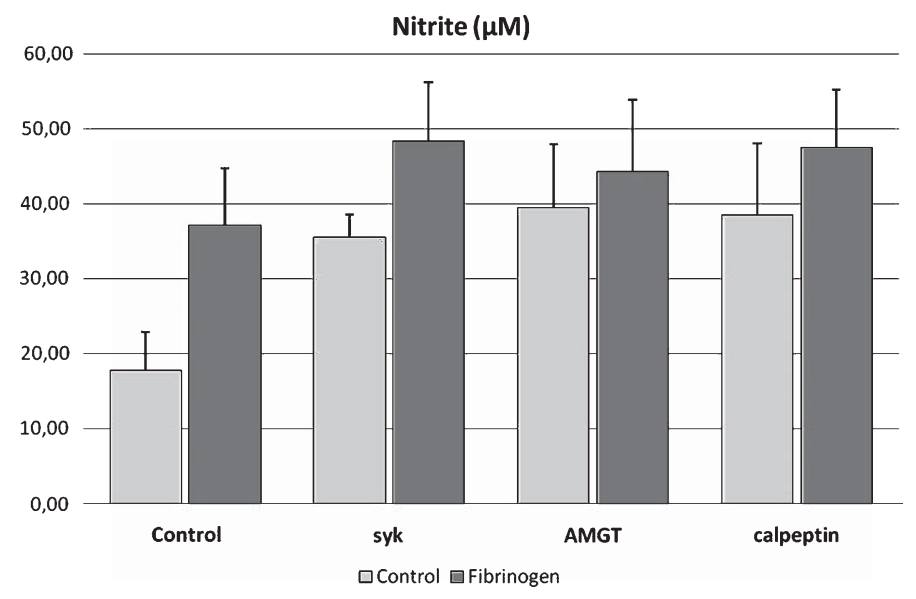

Fig. 2. Changes in nitrite levels in erythrocyte suspensions incubated with high fibrinogen and band 3 modulators. Values are mean $\pm \mathrm{SD}(n=15)$.

In the presence of additional fibrinogen, no changes were observed as to the nitric oxide levels on the dependence of band 3 modulators when compared with those values obtained in its absence. The following concentrations for NO were disclosed: $1.95 \pm 0.36 \mathrm{nM}$ in the presence of Syk inhibitor $10 \mu \mathrm{M}$ ( $p=0.20$, against Syk inhibitor aliquot), (ii) $1.99 \pm 0.27 \mathrm{nM}$ in the presence of AMGT $10 \mu \mathrm{M}(p=0.43$, against AMGT aliquot), and (iii) $2.57 \pm 0.62 \mathrm{nM}$ in the presence of calpeptin $10 \mu \mathrm{M}(p=0.25$, against calpeptin aliquot).

\subsection{Effects of in vitro hyperfibrinogenemia and band 3 phosphorylation/dephosphorylation effectors on erythrocyte nitrite levels (Fig. 2)}

There was a statistically significant increase in nitrite levels of erythrocyte suspensions, in the presence of fibrinogen and all band 3 phosphorylation degree modulators, when compared with both control aliquots and erythrocytes suspensions without additional fibrinogen. 
To specify, in the presence of hyperfibrinogenemia (mean \pm SD: $480.6 \pm 114 \mathrm{mg} / \mathrm{dL}$ ) the levels for nitrite accounted for $37.10 \pm 7.59 \mu \mathrm{M}$, when compared with the control aliquots without fibrinogen $(17.70 \pm 5.18 \mu \mathrm{M}, p<0.00001)$. In the presence of band 3 effectors the following nitrite levels were disclosed: (i) $35.55 \pm 3.02 \mu \mathrm{M}$ with Syk inhibitor (10 $\mu \mathrm{M} p=0.00002$, against control), (ii) $39.45 \pm 8.51 \mu \mathrm{M}$ with AMGT $10 \mu \mathrm{M}$ ( $p=0.00004$, against control), and (iii) $38.50 \pm 9.51 \mu \mathrm{M}$ with calpeptin $10 \mu \mathrm{M}$ $(p=0.0001$, against control).

In the presence of additional fibrinogen, the following concentrations for nitrite were obtained: $48.35 \pm 7.79 \mu \mathrm{M}$ in the presence of Syk inhibitor $10 \mu \mathrm{M}(p=0.001$, against Syk inhibitor $10 \mu \mathrm{M}$ aliquot $)$, (ii) $44.30 \pm 9.54 \mu \mathrm{M}$ in the presence of AMGT $10 \mu \mathrm{M}(p=0.038$, against AMGT10 $\mu \mathrm{M}$ aliquot), and (iii) $47.55 \pm 7.64 \mu \mathrm{M}$ in the presence of calpeptin $10 \mu \mathrm{M}(p=0.066$, against calpeptin $10 \mu \mathrm{M}$ aliquot).

\subsection{Effects of in vitro hyperfibrinogenemia and band 3 phosphorylation/dephosphorylation effectors on erythrocyte nitrate levels (Fig. 3)}

A statistically significant increase in nitrate levels was observed in the presence of fibrinogen and all band 3 phosphorylation degree modulators, when compared with both control aliquots and erythrocytes suspensions without fibrinogen.

To specify, in the presence of hyperfibrinogenemia, (mean \pm SD: $480.6 \pm 114 \mathrm{mg} / \mathrm{dL}$ ) the levels for nitrate accounted for $35.10 \pm 10.19 \mu \mathrm{M}$, when compared with the control aliquots without fibrinogen $(18.95 \pm 4.98 \mu \mathrm{M}, p<0.0005)$. In the presence of band 3 effectors the following nitrite levels were disclosed: (i) $34.10 \pm 6.0 \mu \mathrm{M}$ with Syk inhibitor $10 \mu \mathrm{M}$ ( $p=0.0003$, against control), (ii) $38.85 \pm 5.79 \mu \mathrm{M}$ with AMGT $10 \mu \mathrm{M}(p<0.00001$, against control), and (iii) $37.65 \pm 9.65 \mu \mathrm{M}$ with calpeptin $10 \mu \mathrm{M}$ $(p=0.0002$, against control).

In the presence of additional fibrinogen, the following concentrations for nitrate were obtained: $46.65 \pm 10.82 \mu \mathrm{M}$ in the presence of Syk inhibitor $10 \mu \mathrm{M}(p=0.001$, against Syk inhibitor aliquot $)$, (ii) $42.95 \pm 9.5 \mu \mathrm{M}$ in the presence of AMGT $10 \mu \mathrm{M}$ ( $p=0.10$, against AMGT aliquot), and (iii) $48.84 \pm 7.71 \mu \mathrm{M}$ in the presence of calpeptin $10 \mu \mathrm{M}(p=0.02$, against calpeptin aliquot $)$.

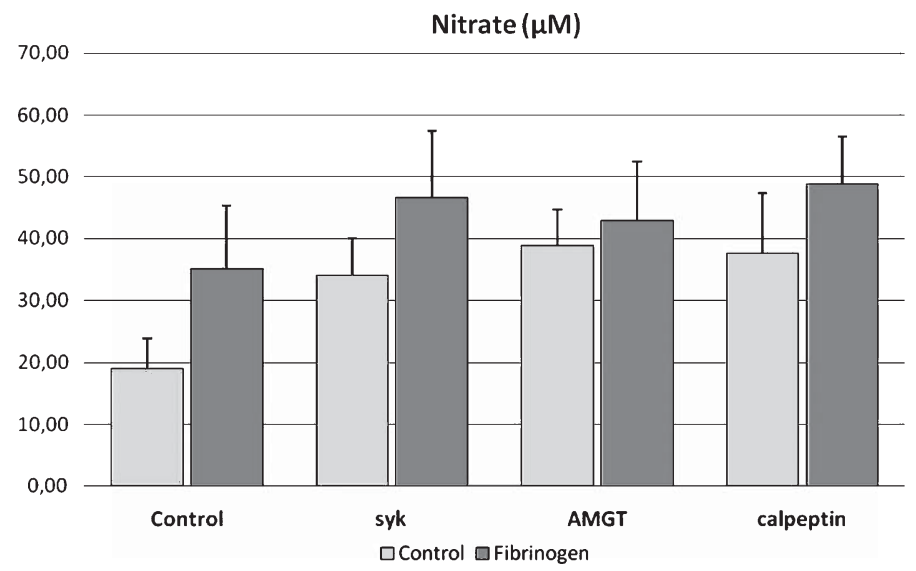

Fig. 3. Changes in nitrate levels in erythrocyte suspensions incubated with high fibrinogen and band 3 modulators. Values are mean $\pm \mathrm{SD}(n=15)$. 


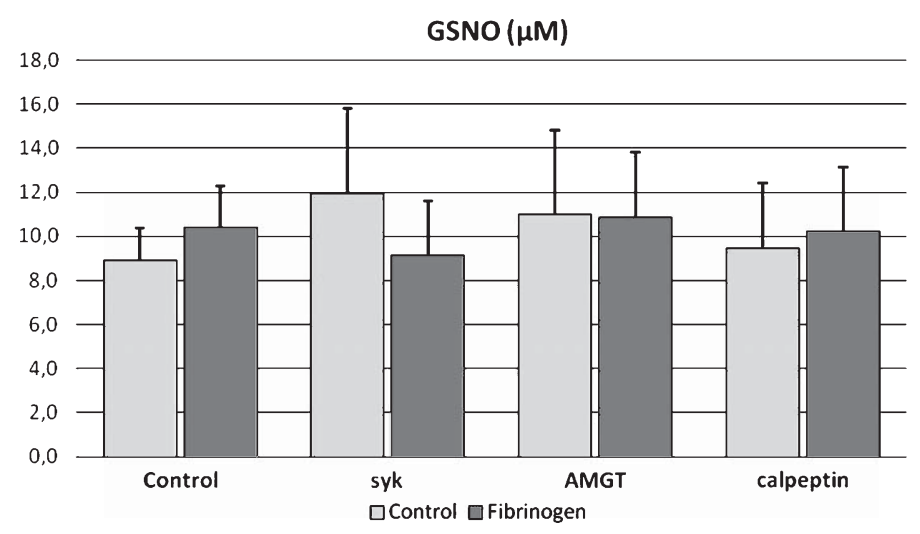

Fig. 4. Effects of high fibrinogen and band 3 modulators concentrations on S-nitrosoglutathione levels in erythrocyte suspensions. Values are mean $\pm \operatorname{SD}(n=15)$.

\subsection{Effects of in vitro hyperfibrinogenemia and band 3 phosphorylation/dephosphorylation effectors on erythrocyte S-nitrosoglutathione (Fig. 4)}

In the presence of hyperfibrinogenemia (mean $\pm \mathrm{SD}: 480.6 \pm 114 \mathrm{mg} / \mathrm{dL}$ ), the levels for GSNO accounted for $10.41 \pm 1.85 \mathrm{mg} / \mathrm{dL}$, when compared with the control aliquots without fibrinogen $(8.91 \pm 1.44 \mathrm{mg} / \mathrm{dL}, p=0.08)$. In the presence of band 3 effectors the following GSNO levels were disclosed: (i) $11.96 \pm 3.86 \mathrm{mg} / \mathrm{dL}$ with Syk inhibitor $10 \mu \mathrm{M}$ ( $p=0.06$, against control), (ii) $10.97 \pm 3.82 \mathrm{mg} / \mathrm{dL}$ with AMGT $10 \mu \mathrm{M}$ ( $p=13$, against control), and (iii) $9.45 \pm 2.96 \mathrm{mg} / \mathrm{dL}$ with calpeptin $10 \mu \mathrm{M}(p=0.61$, against control).

In the presence of additional fibrinogen, the following concentrations for GSNO were obtained: $9.13 \pm 2.47 \mathrm{mg} / \mathrm{dL}$ in the presence of Syk inhibitor $10 \mu \mathrm{M}(p=0.04$, against Syk inhibitor $10 \mu \mathrm{M}$ aliquot $)$, (ii) $10.87 \pm 2.95 \mathrm{mg} / \mathrm{dL}$ in the presence of AMGT $10 \mu \mathrm{M}(p=0.95$, against AMGT $10 \mu \mathrm{M}$ aliquot), and (iii) $10.21 \pm 2.95 \mathrm{mg} / \mathrm{dL}$ in the presence of calpeptin $10 \mu \mathrm{M}(p=0.55$, against calpeptin $10 \mu \mathrm{M}$ aliquot $)$.

\section{Discussion}

To the best of our knowledge, this is the first study to explore an association between fibrinogen and the degree of band 3 phosphorylation on the modulation of erythrocyte nitric oxide efflux and mobilization. We demonstrated that increasing fibrinogen concentrations (hyperfibrinogenemia) is able to module the erythrocyte NO mobilization through a signalling pathway that is dependent on the band 3 phosphorylation status. With high fibrinogen concentrations present in the experimental medium the main findings were an increase in the nitric oxide oxidation molecules, such as nitrite and nitrate, and higher levels of a NO scavenger such as GSNO, along with unaltered NO efflux. These results may be explained by the intra-erythrocyte reactions from NO well-known reservoir molecules and via metabolic reactions, as a way to redistribute NO-derived molecules [19]. For example, the described reaction between GSNO and desoxygenated hemoglobin yielding glutathione, methahemoglobin and NO [37], seems not to be favoured in the presence of high fibrinogen concentrations.

Furthermore, it has been formerly showed by others that the erythrocyte NO flux is mediated by band 3 protein, with no efflux occurring when methemoglobin and deoxygenated hemoglobin bind to the 
cytoskeleton [19]. In view of this, a signal transduction mechanism associating plasma fibrinogen with band 3 protein may be hypothesized in order to explain the lower erythrocyte ability to release NO, in conjunction with the increased intra-erythrocyte amount of nitrite, nitrate and GSNO.

The results of the present study evidenced that phosphorylated (in the presence of calpeptin) or desphosphorylated band 3 (presence of Syk inhibitor or AMGT) significantly increase the erythrocyte efflux, nitrites and nitrates levels but those values are not changed by the presence of high fibrinogen concentrations. The slight increase on GSNO in the presence of Syk inibitor was not verified either with AMGT or calpeptin. However, at high fibrinogen concentrations we observed a slight decrease in GSNO only in the presence of Syk inhibitor, thereby suggesting an antagonism effect due to the external fibrinogen at the erythrocyte membrane. A significantly increase in the NO efflux was verified with high fibrinogen concentration and phosphorylated band 3 protein (presence of calpeptine). Thus, these results document molecular dependence between fibrinogen signalling and the degree of band 3 phosphorylation on the erythrocyte NO mobilization. We may then raise the hypothesis that, with regard to the fibrinogen antioxidant properties, changes in NO metabolism documented in our study might be associated with a redox state induced by fibrinogen in a similar way as dithiothreitol, as previously showed by us [24].

Overall, we can therefore assume that the presence of fibrinogen interferes with erythrocyte NO efflux and mobilization as well as the status of band 3 phosphorylation, but together they seem to have any interaction. In this context, we have additionally documented that acetylcholine is able to increase the erythrocyte NO efflux and modulate signal transduction pathways influenced by the degree of band 3 phosphorylation [8].

At the erythrocyte membrane, band 3 proteins are linked with cytoskeleton protein 4.2 which in turn is associated with the Rh macrocomplex, in which CD47 is one of the components. Any alterations in band 3 conformation induced by phosphorylation or desphorylation may exert an impact on the erythrocyte membrane structure [13].

We have verified a binding between fibrinogen and CD47 [35] which may bring a new explanation for the purported fibrinogen effects on NO efflux and mobilization, based on potential conformational changes induced on these two macro complexes proteins of the erythrocyte membrane. For instance, these possible changes in protein conformation are sufficient to perturb the band 3 conformations obtained by strong covalent bridging of inorganic phosphate molecules or ligation breakdown. On the contrary, when the degree of band 3 phosphorylation was not disturbed, fibrinogen was able to maintain the NO efflux by a still unknown mechanism. As mentioned above, this has been previously documented by us at physiological fibrinogen concentrations and presently in an experimental model of hyperfibrinogenemia. We may then raise the hypothesis that the band 3 phosphorylation-dependent mechanism of NO efflux exists under conditions of external fibrinogen binding to red blood cells.

It is well documented that when red blood cells are stored, this condition depletes them from Snitrosylated hemoglobin [22]. This modification may be responsible for the impaired ability of transfused blood to deliver oxygen [33]. However, our results may be considered as an approach to be followed in blood storage for transfusion, which may impair the progress of band 3 protein modifications $[3,14,31$, 41].

The precise mechanisms by which plasma fibrinogen interacts with the erythrocyte membrane in absence and presence of band 3 modulators of its phosphorylation, to mobilize NO from store molecules into its oxidative metabolites (and vice-versa), are still vague.

Further studies are compulsory to deepen this topic of major impact in human inflammatory conditions and blood storage. 


\section{Acknowledgments}

This study was supported by grants from the FCT - Fundação para a Ciência e a Tecnologia (project reference PTDC/SAU-OSM/73449/2006).

\section{References}

[1] Ethical guidelines for publication in clinical hemorheology and microcirculation, Clin Hemorheol Microcirc 44(1) (2010), $1-2$.

[2] O.K. Baskurt, O. Yalcin and H.J. Meiselman, Hemorheology and vascular control mechanisms, Clin Hemorheol Microcirc 30(3-4) (2004), 169-178.

[3] J.C.G.M.G. Bosman, M. Stappers and V.M.J. Novotny, Changes in band 3 structure as determinants of erythrocyte integrity during storage and survival after transfusion, Blood Transfus 8 (Suppl 3) s48-s52.

[4] A.M. Brunati, L. Bordin, G. Clari, P. James, M. Quadroni, E. Baritono, L.A. Pinna and A. Donella-Deana, Sequential phosphorylation of protein band 3 by Syk and Lyn tyrosine kinases in intact human erythrocytes: Identification of primary and secondary phosphorylation sites, Blood 96 (2000), 1550-1557.

[5] G. Caimi, B. Canino and R.L. Presti, Behaviour of nitric oxide metabolites in unprofessional athletes before and after a cardiopulmonary test, Clin Hemorheol Microcirc 44(4) (2010), 283-286.

[6] G. Caimi, G. Mulè, E. Hopps, C. Carollo and R. Lo Presti, Nitric oxide metabolites and oxidative stress in mild essential hypertension, Clin Hemorheol Microcirc 46(4) (2010), 321-325.

[7] F.A. Carvalho, J.P. Almeida, I.O. Fernandes, T. Freitas-Santos and C. Saldanha, Non-neuronal cholinergic system and signal transduction pathways mediated by band 3 in red blood cells, Clin Hemorheol Microcirc 40 (2008), 207-227.

[8] F.A. Carvalho, A.V. Maria, J.M. Braz-Nogueira, J. Guerra, J. Martins-Silva and C. Saldanha, The relation between the erythrocyte nitric oxide and hemorheological parameters, Clin Hemorheol Microcirc 35 (2006), 341-347.

[9] F.A. Carvalho, J. Martins-Silva and C. Saldanha, Amperometric measurements of nitric oxide in erythrocytes, Biosens Bioelectron 20 (2004), 505-508.

[10] F.A. Carvalho, R. Mesquita, J. Martins-Silva and C. Saldanha, Acetylcholine and choline effects on erythrocyte nitrite and nitrate J levels, J Appl Toxicol 24 (2004), 419-427.

[11] P. Connes, S. Frank, C. Martin, S. Shin, E. Aufradet, S. Sunoo, B. Klara, E. Raynaud de Mauverger, M. Romana, L. Messonnier, J. Kang, E. Varlet-Marie, L. Feasson, M.D. Hardy-Dessources, B. Wilhelm and J.F. Brun, New fundamental and applied mechanisms in exercise hemorheology, Clin Hemorheol Microcirc 45(2-4) (2010), 131-141.

[12] J.A. Cook, S.Y. Kim, D. Teague, M.C. Krishna, R. Pacelli, J.B. Mitchell, Y. Vodovotz, N.R.W.D. Christodoulou, A.M. Miles, M.B. Grisham and D.A.Wink, Convenient colorimetric and fluorometric assays for S-nitrosothiols, Anal Biochem 238 (1996), 150-158.

[13] K.N. Dahl, C.M. Westhoff and D.E. Discher, Fractional attachment of CD47 (IAP) to the erythrocyte cytoskeleton and visual colocalization with Rh protein complexes, Blood 101 (2003), 1194-1199.

[14] E. Farges, R. Grebe and M. Baumann, Viscoelastic and biochemical properties of erythrocytes during storage with SAG-M at +4 degrees C, Clin Hemorheol Microcirc 27(1) (2002), 1-11.

[15] G. Grieninger, K.M. Hertzberg and J. Pindyck, Fibrinogen synthesis in serum-free hepatocyte cultures: Stimulation by glucocorticoids, Proc Natl Acad Sci U S A 75 (1978), 5506-5510.

[16] S.S. Gross and P. Lane, Physiological reactions of nitric oxide and hemoglobin: A radical rethink, Proc Natl Acad Sci U S A 96 (1999), 9967-9969.

[17] S. Grotti and T. Gori, Endothelium, ischemia and the good side of oxygen free radicals, Clin Hemorheol Microcirc 39(1-4) (2008), 197-203.

[18] J. Guevara, I. Iwanejko, A. Dembińska-Kieć, J. Pankiewicz, A. Wanat, P. Anna, I. Gołabek, S. Bartuś, M. MalczewskaMalec and A. Szczudlik, Determination of nitrite/nitrate in human biological material by the simple Griess reaction, Clin Chim Acta 274 (1998), 177-188.

[19] K.T. Huang, T.H. Han, D.R. Hyduke, M.W. Vaughn, H. Van Herle, T.W. Hein, C. Zhang, L. Kuo and J.C. Liao, Modulation of nitric oxide bioavailability by erythrocytes, Proc Natl Acad Sci U S A 98 (2001), 11771-11776. 
[20] F. Jung, From hemorheology to microcirculation and regenerative medicine: Fåhraeus Lecture 2009, Clin Hemorheol Microcirc 45(2-4) (2010), 79-99.

[21] W.B. Kannel, P.A. Wolf, W.P. Castelli and R.B. D’Agostino, Fibrinogen and risk of cardiovascular disease, J Am Med Ass 258 (1987), 1183-1186.

[22] D.B. Kim-Shapiro, J. Lee and M.T. Gladwin, Storage lesion: Role of red blood cell breakdown, Transfusion 51 (2011), 844-851.

[23] A. Liuni, M.C. Luca, T. Gori and J.D. Parker, The endothelial-protective effects of HMG-CoA reductase inhibition in the setting of ischemia and reperfusion injury, Clin Hemorheol Microcirc 45(2-4) (2010), 161-167.

[24] J.P. Lopes de Almeida, F.A. Carvalho, A.S. Silva-Herdade, T. Santos-Freitas and C. Saldanha, Redox thiol status plays a central role in the mobilization and metabolism of nitric oxide in human red blood cells, Cell Biol Int 33 (2009), 268-275.

[25] A. Marossy, P. Svorc, I. Kron and S. Gresová, Circulation hemorheology, Clin Hemorheol Microcirc 42(4) (2009), $239-258$.

[26] S. Muxel, F. Fasola, M.C. Radmacher, A. Jabs, T. Münzel and T. Gori, Endothelial functions: Translating theory into clinical application, Clin Hemorheol Microcirc 45(2-4) (2010), 109-115.

[27] J.R. Pawloski, D.T. Hess and J.S. Stamler, Export by red blood cells of nitric oxide bioactivity, Nature 409 (2001), $622-626$.

[28] J.R. Pawloski and J.S. Stamler, Nitric oxide in RBCs, Transfusion 42 (2002), 1603-1609.

[29] M.J. Pearson and H.H. Lipowsky, Effect of fibrinogen on leukocyte margination and adhesion in postcapillary venules, Microcirculation 11 (2004), 295-306.

[30] K. Perampaladas, J.D. Parker and T. Gori, The impact of rosiglitazone on nitric oxide bioavailability and endothelial function, Clin Hemorheol Microcirc 45(2-4) (2010), 325-328.

[31] G. Pindur, D. Seiffge, R.P. Franke, U.T. Seyfert and F. Jung, (Quality of erythrocyte concentrates in additive solutionsevaluation with hemorrheological methods), Anasthesiol Intensivmed Notfallmed Schmerzther 36(Suppl 1) (2001), S6-S10.

[32] M.W. Ramping, The great divide: From viscometer to vasculature, Clin Hemorheol Microcirc 39(1-4) (2008), 9-20.

[33] J.D. Reynolds, D.T. Hess and J.S. Stamler, The transfusion problem: Role of aberrant S-nitrosylation, Transfusion 51 (2011), 852-858.

[34] R. Roststein, T. Landau, A. Twig, A. Rubinstein, M. Koffler, D. Justo, D. Constantiner, D. Zeltzer, I. Shapira, T. Mardi, Y. Goldin and S. Berliner, The erythrocyte adhesiveness/aggregation test (EAAT). A mew Biomarker to reveal the presence of low grade subclinical smoldering inflammation in individuald with atherosclerotic risk factors, Atherosclerosis 165 (2002), 343-351.

[35] C. Saldanha, V. Almeida, H.S. Rosário, J.P. Almeida and S. Oliveira, Erythrocyte membrane binding fibrinogen. Abstracts Book of the 9th World Congress for Microcirculation, Paris, 2010.

[36] L. Sargento, C. Saldanha, J. Monteiro, C. Perdigão and J.M. Silva, Long-term prognostic value of protein C activity, erythrocyte aggregation and membrane fluidity in transmural myocardial infarction, Thromb Haemost 94(2) (2005), 380-388.

[37] N.Y. Spencer, H. Zeng, R.P. Patel and N. Hogg, Reaction of S-nitrosoglutathione with the heme group of deoxyhemoglobin, J Biol Chem 275 (2000), 36562-36567.

[38] M.M. Tarpey and I. Fridovich, Methods of detection of vascular reactive species: Nitric oxide, Superoxide, Hydrogen peroxide, and Peroxynitrite, Circ Res 89 (2001), 224-236.

[39] J. Tripette, P. Connes, E. Beltan, T. Chalabi, L. Marlin, R. Chout, O.K. Baskurt, O. Hue and M.D. Hardy-Dessources, Red blood cell deformability and aggregation, cell adhesion molecules, oxidative stress and nitric oxide markers after a short term, submaximal, exercise in sickle cell trait carriers, Clin Hemorheol Microcirc 45(1) (2010), 39-52.

[40] P. Ulker, H.J. Meiselman and O.K. Baskurt, Nitric oxide generation in red blood cells induced by mechanical stress, Clin Hemorheol Microcirc 45(2-4) (2010), 169-175.

[41] M. Uyuklu, M. Cengiz, P. Ulker, T. Hever, J. Tripette, P. Connes, N. Nemeth, H.J. Meiselman and O.K. Baskurt, Effects of storage duration and temperature of human blood on red cell deformability and aggregation, Clin Hemorheol Microcirc 41(4) (2009), 269-278.

[42] Z. Yu, J.P. Ouyang and Y.P. Li, Dexamethasone attenuated endotoxin-induced acute lung injury through inhibiting expression of inducible nitric oxide synthase, Clin Hemorheol Microcirc 41(2) (2009), 117-125. 